\title{
APLICAÇÃO DE REATORES ANAERÓBIOS OPERADOS EM BATELADAS SEQUENCIAIS COM FILME FIXO NO TRATAMENTO DE ÁGUA RESIDUARIA INDUSTRIAL RICA EM SULFATO
}

\section{APPLICATION OF THE ANAEROBIC SEQUENCING BATCH REACTORS WITH FIXED FILM IN THE TREATMENT OF INDUSTRIAL WASTEWATER CONTAINING HIGH SULFATE CONCENTRATION}

\section{Arnaldo Sarti ${ }^{1}$; Eugênio Foresti ${ }^{2}$}

${ }^{1}$ Escola de Engenharia de São Carlos - USP (Departamento de Hidráulica e Saneamento)

Av. João Dagnone, № 1100, Santa Angelina, 13.563-120 - São Carlos (SP), Fone (16) 3373-8357, e-mail: arnaldosarti@gmail.com

${ }^{2}$ Escola de Engenharia de São Carlos - USP (Departamento de Hidráulica e Saneamento)

Av. João Dagnone, № 1100, Santa Angelina, 13.563-120 - São Carlos (SP), Fone (16) 3373-8358, e-mail: eforesti@sc.usp.br

\section{RESUMO}

Este trabalho apresenta e discute o potencial de uso de reatores anaeróbios operados em bateladas seqüenciais com biomassa imobilizada (ASBBR), em escala piloto, no tratamento de água residuária industrial contendo elevadas concentrações de sulfato. No ASBBR, com volume total de $1,2 \mathrm{~m}^{3}$, preenchido com carvão mineral (meio suporte) foram aplicadas cargas de sulfato de 0,15 a $1,90 \mathrm{~kg} /$ ciclo com duração de ciclo de $48 \mathrm{~h}$, correspondendo, respectivamente, às concentrações de sulfato no afluente de 0,25 a 3,0 g.L $\mathrm{L}^{-1}$. O esgoto sanitário e etanol foram usados como doadores de elétrons para a redução do sulfato. As eficiências médias na redução de sulfato ficaram entre 88 e $92 \%$ nos 92 ciclos operacionais. Como pós-tratamento dos efluentes gerados pela redução de sulfato empregou-se outro reator ASBBR com volume total de 385,0 L e mesmo meio suporte para imobilização da biomassa, mas com diferente granulometria. A remoção média de DQO (valor médio afluente: 1450 mg. $\left.\mathrm{L}^{-1}\right)$ foi de $88 \%$, enquanto as concentrações de sulfetos totais $\left(\mathrm{H}_{2} \mathrm{~S}, \mathrm{HS}^{-}, \mathrm{S}^{2-}\right)$ 
mantiveram-se entre a faixa de 41 e $71 \mathrm{mg} . \mathrm{L}^{-1}$ nos 35 ciclos operacionais de $48 \mathrm{~h}$. Os resultados obtidos permitiram concluir que o uso de reatores ASBBR constitui-se em potencial alternativa tanto para a remoção de sulfatos, como no pós-tratamento dos efluentes gerados.

PALAVRAS-CHAVE: Sulfato. Sulfeto. Tratamento anaeróbio. Carvão mineral.

\section{ABSTRACT}

This paper presents and discusses the potential for use of pilot-scale anaerobic sequencing batch biofilm reactors (ASBBR) for the treatment of industrial wastewater containing high sulfate concentration. The pilot-scale ASBBR reactor (total volume $=1.2 \mathrm{~m}^{3}$ ) containing biomass immobilized in inert support (mineral coal) was operated at sulfate loading rates varying from 0.15 to $1.90 \mathrm{kgSO}_{4}^{-2} /$ cycle (cycle of $48 \mathrm{~h}$ ) corresponding to sulfate concentrations of 0.25 to $3.0 \mathrm{gSO}_{4}^{-2} \cdot \mathrm{L}^{-1}$. Domestic sewage and ethanol were utilized as electron donors for sulfate reduction. The mean sulfate removal efficiencies remained in the range of 88 to $92 \%$ in the several sulfate concentrations obtained from 92 operational cycles. As post-treatment unit for the generated effluents by the sulfate reduction was used another reactor ASBBR in pilot-scale (total volume $=385,0 \quad$ L) with same inert support for biomass immobilization and different granulometry. The mean COD removal (mean influent $\left.=1450 \mathrm{mg} \cdot \mathrm{L}^{-1}\right)$ achieved $88 \%$ and total sulfide concentrations $\left(\mathrm{H}_{2} \mathrm{~S}, \mathrm{HS}^{-}, \mathrm{S}^{2-}\right)$ remained in the range of 41 to $71 \mathrm{mg} . \mathrm{L}^{-1}$ during the 35 operational cycles of $48 \mathrm{~h}$. The results demonstrated that the use of ASBBR reactors is an alternative potential for the sulfate removal and as post-treatment of generated effluent.

KEYWORDS: Sulfate. Sulfide. Anaerobic treatment. Mineral Coal

\section{INTRODUÇÃO}

O Departamento Nacional de Produção Mineral (DNPM) relata que, em 2005, o consumo de enxofre no país decorre, principalmente, de sua aplicação na agricultura em forma de fertilizantes e nas indústrias químicas para produção de ácido sulfúrico. O Brasil importou aproximadamente 2,0 milhões de toneladas de compostos de enxofre, entre bens primários e compostos industrializados (ácido sulfúrico, enxofre sublimado, pirita, e outros), correspondente ao dispêndio de US $\$ 108,6$ milhões e exportou 6,0 mil toneladas de enxofre como bens primários, como a pirita e compostos industrializados (por exemplo, ácido sulfúrico). Outros importantes setores industriais utilizam compostos de enxofre, tais como, para a fabricação de pigmentos inorgânicos, celulose e papel, borracha, bissulfeto de carbono, explosivos, açúcar e cosméticos (COLLERAN et al., 1994). Portanto, 
grande parte do enxofre importado, se não for tratado, tem como destino os cursos d'água na forma de compostos oxidados de enxofre, principalmente o sulfato.

As descargas indiscriminadas de águas residuárias contendo sulfato, em corpos receptores, podem prejudicar a qualidade das águas e também interferir no ciclo natural do enxofre. Nas zonas anaeróbias, o íon sulfato é reduzido a sulfeto de hidrogênio, aumentando a demanda química de oxigênio. Dessa forma, o lançamento direto de sulfato em um corpo de água compromete o processo natural de autodepuração. Considerando os problemas que o sulfato pode causar se lançado no ambiente, os órgãos de controle ambiental têm exigido que as fontes emissoras reduzam as concentrações desse ânion nas águas residuárias. Vale mencionar que a Resolução CONAMA 357 de 17 de março de 2005, não faz menção a valores máximos de sulfatos, mas apresenta valor máximo de sulfetos $\left(1,0 \mathrm{mg} . \mathrm{L}^{-1}\right)$. No Estado de São Paulo, a Lei no 997 de 31 de maio de 1976, regulamentada pelo Decreto Estadual 8468 de 1976, no Artigo 19-A, estabelece a máxima concentração de sulfato de $1000 \mathrm{mg} . \mathrm{L}^{-1}$ em efluentes líquidos lançados nos corpos receptores (SARTI et al., 2008).

No Brasil, os processos físicos e químicos utilizados, para remoção de sulfato incluem desde alternativas de menor custo, como precipitação com sais de cálcio, a processos mais caros como osmose reversa, eletrodiálise e nanofiltração. Processos de precipitação química resultam em grande quantidade de resíduos sólidos devido à quantidade de reagente a ser empregada. Esses resíduos devem ser adequadamente dispostos, incorporando custos para classificação, manuseio e destinação (SILVA et al., 2002).

No processo de osmose reversa, a concentração de sulfato limita a vida útil da membrana devido à precipitação deste ânion, além de gerar rejeito na forma de salmoura (SILVA et al., 2002). Os demais processos têm o custo proporcional à concentração de sulfato, de forma que altas concentrações chegam a inviabilizá-los. Neste caso, para o tratamento de águas residuárias com elevadas concentrações de sulfato, a remoção biológica apresenta-se como alternativa aos processos físicoquímicos. Dependendo da concentração de sulfato na água residuária e das restrições legais para emissão do ânion, pode ser interessante a união de processos físicos e químicos aos processos biotecnológicos.

$\mathrm{Na}$ última década, várias alternativas biotecnológicas têm sido desenvolvidas para a remoção de ânions de enxofre oxidados $\left(\mathrm{SO}_{3}{ }^{2-}, \mathrm{HSO}_{3}{ }^{-}, \mathrm{S}_{2} \mathrm{O}_{3}{ }^{2-}, \mathrm{SO}_{4}{ }^{2-}\right.$, e outros) de efluentes líquidos industriais. Primeiro, os óxidos de enxofre são transformados em sulfeto $\left[\mathrm{H}_{2} \mathrm{~S}(\mathrm{~g})+\mathrm{H}_{2} \mathrm{~S}(\mathrm{aq})+\mathrm{HS}^{-}\right]$em ambiente anaeróbio. Os ânions de enxofre reduzidos são subseqüentemente re-oxidados para enxofre elementar $\left(S^{0}\right)$, o qual pode ser recuperado (LENS et al. 1998). Atualmente, os principais reatores biológicos utilizados comercialmente e em escala industrial são o FSBR (Falling Sludge Bed Reactor) e o UASB (Upflow Anaerobic Sludge Bed) que correspondem ao Biosure Process (África do Sul) e ao Pacques Process (Holanda), respectivamente (HULSHOFF-POL et al., 2001). 
Estes processos são de grande interesse na remoção de compostos oxidados de enxofre para as águas residuárias que não contêm doador de elétrons ou fonte de carbono (ou contêm em quantidade insuficiente) para a completa redução do sulfato. Para essas águas residuárias, é requerida a adição de um doador de elétrons apropriado. A seleção do doador de elétrons depende do custo do composto adicionado por unidade de sulfato reduzido e da poluição residual decorrente da degradação parcial do composto adicionado, a qual deve ser baixa ou facilmente removível. Com base neste último critério, compostos orgânicos simples (etanol, metanol) ou gás de síntese (mistura de $\mathrm{H}_{2}, \mathrm{CO}$ e $\mathrm{CO}_{2}$ ) são preferíveis em relação a substratos orgânicos mais complexos (VAN HOUTEN et al.,1996).

A partir da presença de fonte orgânica (doadores de elétrons), a relação $\mathrm{DQO} /\left[\mathrm{SO}_{4}{ }^{2-}\right]$ é um dos principais fatores para definir a interação entre os microrganismos metanogênicos e os redutores de sulfato (SARTI, 2008). Para o tratamento biológico de sulfato via processo anaeróbio, teoricamente, o valor desta relação deve ser igual a 0,67 . Neste caso, há sulfato suficiente para que toda a fonte de matéria orgânica seja consumida via sufetogenese (LENS et al.,1998). Para situações, onde estes valores são elevados, os processos metanogênicos e sufetogênicos (redução de sulfato) podem ocorrer simultaneamente e abaixo deste, a sufetogênese poder ser a predominante.

No caso específico do uso do etanol para sistemas desenvolvidos com o intuito de redução de sulfato, o maior problema a ser enfrentado, é a produção de quantidade apreciável de ácido acético quando a predominância do processo sulfetogênico está estabelecida (oxidação incompleta). Desta forma existe a presença no efluente de alta concentração de DQO residual, bem como dos sulfetos gerados no processo de redução (NAGPAL et al., 2000). Consequentemente, este efluente originado pela adição de etanol deve ser tratado em unidade subseqüente, onde os sulfetos podem ser parcialmente re-oxidados para enxofre elementar e a DQO residual minimizada, via metanogênese pelo emprego de processo anaeróbio.

O sulfeto pode ser oxidado, empregando-se sistemas de aeração não catalisados ou biocatalisados, eletroquimicamente, ou quimicamente, utilizando-se cloro, ozônio, permanganato de potássio ou peróxido de hidrogênio, com produção de enxofre elementar, tiossulfato ou sulfato, dependendo do $\mathrm{pH}$. Outros métodos considerados convenientes para remoção de sulfeto são: precipitação com íons metálicos e o arraste para a atmosfera do $\mathrm{H}_{2} \mathrm{~S}$. Os consumos de substâncias e de energia, além da geração de resíduos, entretanto, constituem em desvantagens da utilização desses processos (LENS et al., 1998).

O sulfeto de hidrogênio e o dióxido de enxofre podem ser convertidos biologicamente a enxofre elementar (JANSSEN et al., 1998). O enxofre elementar pode ser produzido mediante a oxidação incompleta de sulfeto por Thiobacillus spp., conforme a Equação (1). Sob condições limitadas de oxigênio (Oxigênio Dissolvido $\left.(\mathrm{OD}) \leq 0,1 \mathrm{mg} \cdot \mathrm{L}^{-1}\right), \mathrm{S}^{0}$ é o principal produto final da oxidação do sulfeto. A formação requer quatro vezes menos oxigênio (menor custo energético), comparado com a 
oxidação completa (Equação 2) para o retorno na forma de sulfato. O enxofre elementar $\left(\mathrm{S}^{0}\right)$ biologicamente produzido tem coloração branca ou amarela palha, tem índice de refração maior que o da água e é classificado como líquido amorfo com base em resultados de difração de Raios X.

$$
\begin{array}{ll}
2 \mathrm{HS}^{-}+\mathrm{O}_{2} \rightarrow 2 \mathrm{~S}^{0}+2 \mathrm{OH}^{-} & \Delta \mathrm{G}^{0}=-169,35 \mathrm{~kJ} . \mathrm{mol}\left(\mathrm{HS}^{-}\right)^{-1} \\
2 \mathrm{HS}^{-}+4 \mathrm{O}_{2} \rightarrow 2 \mathrm{SO}_{4}^{-2}+2 \mathrm{H}^{+} & \Delta \mathrm{G}^{0}=-732,58 \mathrm{~kJ} . \mathrm{mol}\left(\mathrm{HS}^{-}\right)^{-1}
\end{array}
$$

Este trabalho apresenta e discute os resultados obtidos por duas unidades ASBBR (Anaerobic Sequencing Batch Biofilm Reactor) com biomassa imobilizada em carvão mineral e recirculação de líquido. Na primeira unidade $\left(1,2 \mathrm{~m}^{3}\right)$, a proposta foi de tratamento via processo anaeróbio do sulfato presente em água residuária industrial e na segunda unidade, o pós-tratamento do efluente gerado no processo de redução de sulfato, pela utilização de etanol com doador de elétrons.

\section{MATERIAL E MÉTODOS}

Água Residuária Industrial

A água residuária rica em sulfato foi proveniente de indústria química que tem, como principal atividade, a fabricação de óleo para acabamento de couros animais, processo este baseado na sulfonação de óleos vegetais (arroz, soja e milho). A reação completa de sulfonação ocorre na presença de ácido sulfúrico $\left(\mathrm{H}_{2} \mathrm{SO}_{4}\right)$ e amônia (líquida) em reator operado em batelada e sob temperatura controlada. No final do processo de sulfonação, ocorre a lavagem do óleo produzido com água, cujo objetivo principal é a eliminação dos ácidos livres. A composição da água residuária de lavagem com alta concentração de sulfato é apresentada na Tabela 1.

Tabela 1- Composição dos principais constituintes da água residuária industrial (20 amostras).

\begin{tabular}{lccc}
\hline Parâmetros & Mínimo & Máximo & Média \\
\hline $\mathrm{pH}$ & 2,30 & 3,20 & - \\
$\mathrm{DQO}_{\text {Total }}\left(\mathrm{g} . \mathrm{L}^{-1}\right)$ & 9,20 & 15,40 & $12,70 \pm 4,1$ \\
$\mathrm{DQO}_{\text {Filtrada }}\left(\mathrm{g} . \mathrm{L}^{-1}\right)$ & 9,11 & 10,90 & $10,60 \pm 1,3$ \\
$\mathrm{NH}_{4}^{+}\left(\mathrm{g} . \mathrm{L}^{-1}\right)$ & 1,30 & 1,80 & $1,50 \pm 0,5$ \\
$\mathrm{SO}_{4}^{-2}\left(\mathrm{~g} . \mathrm{L}^{-1}\right)$ & 180 & 284 & $201 \pm 35$ \\
\hline
\end{tabular}




\section{Reatores ASBBR (Projeto e Operação)}

Nos reatores ASBBR, a agitação foi provida pela recirculação ascendente do líquido por meio de bomba centrifuga cuja canalização de recalque estava conectada a um tubo perfurado situado na base do leito de carvão ao longo do diâmetro do reator. A mesma bomba de recirculação foi empregada para a alimentação dos reatores (afluente). $\mathrm{O}$ tempo de ciclo de $48 \mathrm{~h}$ foi mantido o mesmo, compreendendo as etapas de alimentação $(1 \mathrm{~h})$, reação com recirculação de líquido contínua $(46 \mathrm{~h}) \mathrm{e}$ descarte $(1 \mathrm{~h})$.

$\mathrm{Na}$ Tabela 2 estão apresentadas às características construtivas e operacionais das duas unidades ASBBR em escala-piloto para realização dos ensaios experimentais. Ressalta-se que em ambos os reatores, o volume destinado ao acúmulo de biogás foi reduzido pela necessidade de se manter a tubulação de recirculação sempre completa. Este volume reduzido para acúmulo de biogás é representado por $\mathrm{V}_{\mathrm{A}}$. Portanto, o volume líquido total ou disponível para tratamento por ciclo ou batelada é o somatório de $\mathrm{V}_{\mathrm{L}}$ e $\mathrm{V}_{\mathrm{A}}$. A tubulação de saída do biogás dos reatores foi imersa em recipiente $(100 \mathrm{~L})$ contendo solução alcalina $(\mathrm{NaOH})$.

Tabela 2 - Características construtivas e operacionais dos Reatores ASBBR (projetados)

\begin{tabular}{|c|c|c|}
\hline Características & $\begin{array}{c}\text { ASBBR } \\
\text { (Tratamento de sulfato) }\end{array}$ & $\begin{array}{c}\text { ASBBR } \\
\text { (Pós-Tratamento) }\end{array}$ \\
\hline Material & Fibra de vidro & Fibra de vidro \\
\hline Altura (L) & $2400 \mathrm{~mm}$ & $1800 \mathrm{~mm}$ \\
\hline Diâmetro (D) & $800 \mathrm{~mm}$ & $520 \mathrm{~mm}$ \\
\hline Relação (L/D) & 3,0 & 3,5 \\
\hline *Massa de Carvão Mineral & $520 \mathrm{~kg}(\varepsilon=0,51)$ & $197 \mathrm{~kg}(\varepsilon=0,53)$ \\
\hline Diâmetro Carvão Mineral & 40 a $80 \mathrm{~mm}$ & 20 a $40 \mathrm{~mm}$ \\
\hline Volume Líquido do Leito $\left(\mathrm{V}_{\mathrm{L}}\right)$ & $510 \mathrm{~L}$ & $160 \mathrm{~L}$ \\
\hline Volume Total do Leito $\left(\mathrm{V}_{\mathrm{TL}}\right)$ & $1000 \mathrm{~L}$ & $303 \mathrm{~L}$ \\
\hline Volume Head-space $\left(\mathrm{V}_{\mathrm{H}}\right)$ & $40 \mathrm{~L}$ & $22 \mathrm{~L}$ \\
\hline **Volume Adicional $\left(\mathrm{V}_{\mathrm{A}}\right)$ & $90 \mathrm{~L}$ & $60 \mathrm{~L}$ \\
\hline ***Volume Líquido Total $\left(\mathrm{V}_{\mathrm{A}}+\mathrm{V}_{\mathrm{L}}\right)$ & $600 \mathrm{~L}$ & $220 \mathrm{~L}$ \\
\hline Volume Total do reator $\left(\mathrm{V}_{\mathrm{TL}}+\mathrm{V}_{\mathrm{H}}+\mathrm{V}_{\mathrm{A}}\right)$ & $1200 \mathrm{~L}$ & $385 \mathrm{~L}$ \\
\hline Volume Útil $\left(\mathrm{V}_{\mathrm{TL}}+\mathrm{V}_{\mathrm{A}}\right)$ & $1100 \mathrm{~L}$ & $363 \mathrm{~L}$ \\
\hline Tipo de Agitação & contínua & Contínua \\
\hline Equipamento (agitação) & $\begin{array}{l}\text { bomba centrifuga } \\
\text { (Jacuzzi 5JL15) }\end{array}$ & $\begin{array}{l}\text { bomba centrifuga } \\
\text { (Thebe B12NR) }\end{array}$ \\
\hline Condições de Agitação (Vazões) & 3,3 a $5,3 \mathrm{~m}^{3} \cdot \mathrm{h}^{-1}$ & 0,6 a $1,8 \mathrm{~m}^{3} \cdot \mathrm{h}^{-1}$ \\
\hline $\begin{array}{l}\text { * Porosidade de leito }(\varepsilon)=\mathrm{V}_{\mathrm{A}} / \mathrm{V}_{\mathrm{TL}} \\
* * \text { Nível líquido para sucção da bomba } \\
* * * \text { Volume de líquido a ser tratado }\end{array}$ & & \\
\hline
\end{tabular}


Os reatores foram instalados no Laboratório de Processos Biológicos (Escola de Engenharia de São Carlos-Universidade de São Paulo, São Carlos), que dispõe de sistema de coleta de esgotos sanitários. No experimento com o ASBBR1, o esgoto sanitário foi utilizado para diluir a água residuária industrial rica em sulfato (Tabela 1), a fim de permitir a operação do reator na concentração de sulfato desejada.

O ASBBR1 foi mantido à temperatura ambiente média de $29 \pm 8^{\circ} \mathrm{C}$ ao longo dos 92 ciclos de operação. Durante o experimento, a concentração de sulfato afluente ao reator foi incrementada por meio da diluição em esgoto sanitário, tendo sido aplicadas concentrações de sulfato de 0,$25 ; 0,50 ; 1,0 ; 2,0$ e 3,0 g. $\mathrm{L}^{-1}$. O ASBBR2 foi mantido à temperatura ambiente média de $24 \pm 9{ }^{0} \mathrm{C}$ ao longo dos 35 ciclos de operação.

Na Figura 1 apresenta-se o desenho esquemático das instalações experimentais de tratamento de sulfato (ASBBR1) e de pós-tratamento (ASBBR2) utilizadas na pesquisa.

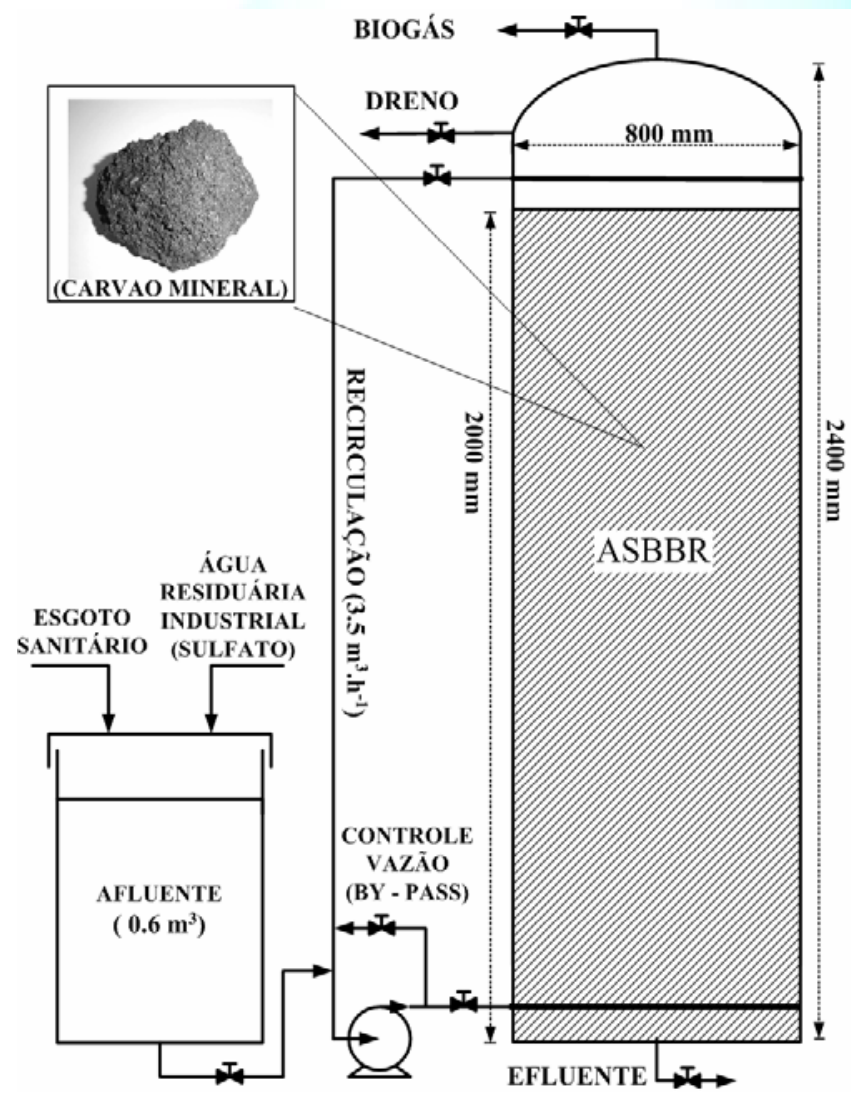

(a)

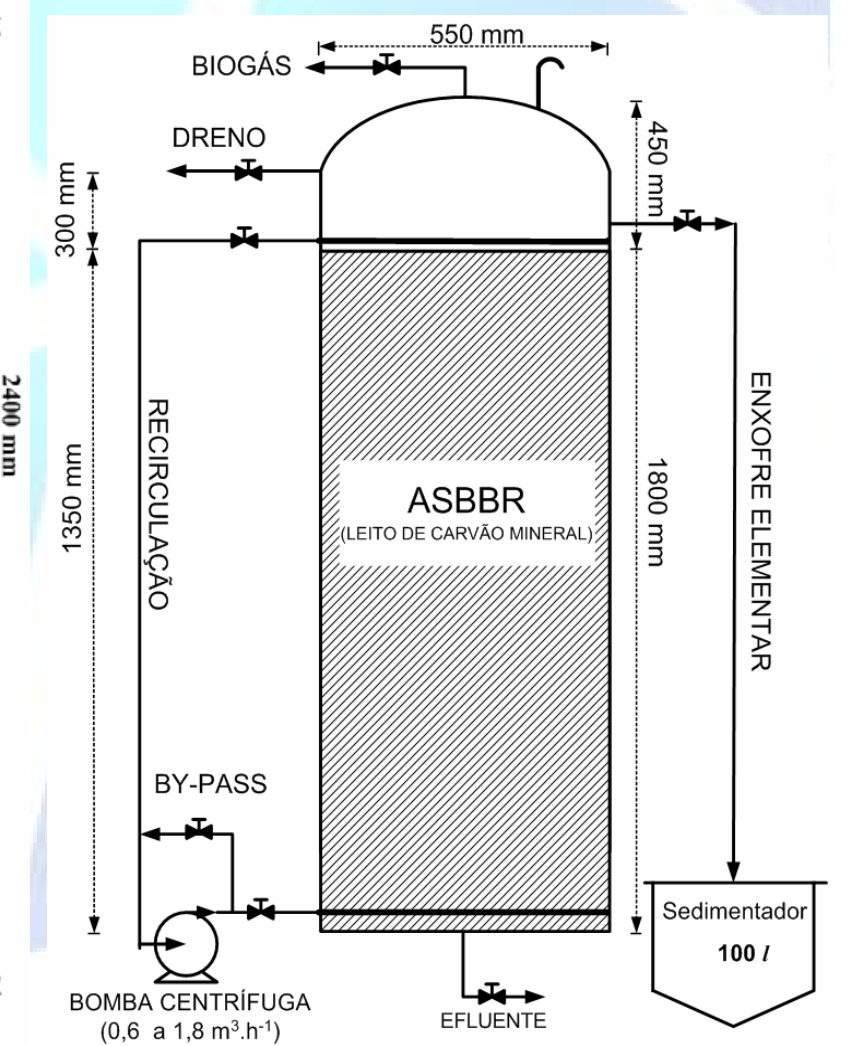

(b)

Figura 1. Desenho esquemático das instalações experimentais (a-ASBBR1 e b-ASBBR2) 
A Tabela 3 apresenta as variáveis operacionais aplicadas ao ASBBR1 nas diferentes etapas experimentais. No reator, foi aplicada inicialmente a carga de sulfato (CS) de $0,15 \mathrm{~kg} /$ ciclo (Etapa I- $0,25 \mathrm{~g} . \mathrm{L}^{-1}$ ), sendo esta incrementada até 1,9 $\mathrm{kg} /$ ciclo (Etapa V- 3,0 g. $\mathrm{L}^{-1}$ ) no ensaio experimental. Salienta-se que, na Etapa I, apenas o esgoto sanitário foi usado como doador de elétrons (Etapa I). A partir da Etapa II $\left(0,30 \mathrm{~kg} /\right.$ ciclo ou $\left.0,5 \mathrm{~g} . \mathrm{L}^{-1}\right)$ foi adicionado, também, o etanol, como fonte suplementar para a redução biológica do sulfato. $\mathrm{O}$ volume adicionado do etanol foi ajustado progressivamente em função da relação $\mathrm{DQO} /\left[\mathrm{SO}_{4}{ }^{2-}\right]$, com intuito de maximizar as eficiências de redução do sulfato (Tabela 3). Não se aplicou estequiometria reacional específica, para portanto, que fosse maximizado o processo de conversão do sulfato e redução do consumo de etanol simultaneamente.

Conforme estabelece a Legislação vigente no Estado de São Paulo, a concentração máxima permitida para efluentes líquidos lançados direta ou indiretamente nos corpos receptores é de $1,0 \mathrm{gSO}_{4}{ }^{2-} \cdot 1^{-1}$. Neste caso, adotaram-se as Etapas I e II $\left(0,25\right.$ e $\left.0,50 \mathrm{gSO}_{4}^{-2} \cdot 1^{-1}\right)$ como sendo de adaptação da biomassa inoculada aderida ao carvão mineral na presença do efluente industrial diluído em esgoto sanitário. Esperava-se que esse período de adaptação fosse demorado, em virtude de se ter que aclimatar a biomassa inoculada, mas ocorreram rápida colonização e adaptação, já que se obtiveram elevados valores de remoção de sulfato e de matéria orgânica desde o inicio de operação (Etapa I). Posteriormente, atingiu-se concentração de sulfato de 3,0 g.L. ${ }^{-1}$ (Etapa V) para verificação da adequação dos resultados em termos de redução de sulfato em função da legislação vigente.

\section{Monitoramento}

O monitoramento dos reatores ASBBRs foi realizado por meio de análises físico-químicas (afluente e efluente) tais como demanda química de oxigênio (DQO) total e filtrada (membrana 1,2 $\mu \mathrm{m}$ ), sólidos suspensos totais (SST) e sólidos suspensos voláteis (SSV) e pH conforme Standard Methods for the Examination of Water and Wastewater (APHA, 1998). As determinações de ácidos voláteis totais (AVT) como ácido acético $\left(\mathrm{H}_{\mathrm{AC}}\right)$ e alcalinidade a bicarbonato $(\mathrm{AB})$ seguiram metodologia descrita por Dillalo e Albertson (1961) e modificada por Ripley et al. (1986), respectivamente. O método de azul de metileno (Standard Methods for the Examination of Water and Wastewater: método 4500-D) foi usado para determinação de sulfetos totais dissolvidos (STD). As concentrações de sulfato foram medidas pelo método turbidimétrico) o uso do reagente $\mathrm{HACH}$ sulfaver. As determinações de potencial redox foram realizadas com o uso eletrodos específicos (YELLOW SPRING-600). 
Tabela 3 - Valores médios das variáveis aplicadas ao reator ASBBR1 nas várias Etapas de operação.

\begin{tabular}{|c|c|c|c|c|c|}
\hline Parâmetros & Etapa I & Etapa II & Etapa III & Etapa IV & Etapa V \\
\hline Número de ciclos & $7(5)$ & $24(16)$ & $21(14)$ & $9(6)$ & $31(20)$ \\
\hline CS (kg/ciclo) & $0,15 \pm 0,02$ & $0,30 \pm 0,03$ & $0,65 \pm 0,07$ & $1,30 \pm 0,14$ & $1,90 \pm 0,13$ \\
\hline $\mathrm{SO}_{4}^{-2}\left(\mathrm{~g} \cdot \mathrm{L}^{-1}\right)$ & $0,25 \pm 0,03$ & $0,50 \pm 0,05$ & $1,08 \pm 0,12$ & $2,16 \pm 0,24$ & $3,12 \pm 0,23$ \\
\hline $\mathrm{DQO}^{*} /\left[\mathrm{SO}_{4}{ }^{-2}\right]$ & $2,13 \pm 0,35$ & $1,89 \pm 0,65$ & $1,77 \pm 0,26$ & $1,64 \pm 0,40$ & $1,50 \pm 0,25$ \\
\hline CO (kg/ciclo) & $0,60 \pm 0,05$ & $0,90 \pm 0,17$ & $1,40 \pm 0,16$ & $2,50 \pm 0,60$ & $3,0 \pm 0,47$ \\
\hline $\mathrm{DQO}_{\text {Total }}\left(\mathrm{g} \cdot \mathrm{L}^{-1}\right)$ & $0,98 \pm 0,08$ & $1,49 \pm 0,29$ & $2,35 \pm 0,27$ & $4,12 \pm 1,0$ & $5,08 \pm 0,78$ \\
\hline $\mathrm{DQO}_{\text {Filtrada }}\left(\mathrm{g} \cdot \mathrm{L}^{-1}\right)$ & $0,54 \pm 0,08$ & $0,93 \pm 0,28$ & $1,89 \pm 0,26$ & $3,57 \pm 1,0$ & $4,63 \pm 0,78$ \\
\hline $\mathrm{AB}\left(\mathrm{mgCaCO}_{3} \cdot \mathrm{L}^{-1}\right)$ & $103 \pm 15$ & $103 \pm 45$ & $93 \pm 19$ & $84 \pm 13$ & $92 \pm 26$ \\
\hline AVT (mgHac.L $\left.{ }^{-1}\right)$ & $67 \pm 11$ & $67 \pm 16$ & $112 \pm 23$ & $96 \pm 11$ & $119 \pm 33$ \\
\hline $\mathrm{pH}$ & 6,9 & 7,0 & 6,7 & 6,9 & 6,7 \\
\hline $\mathrm{SST}\left(\mathrm{mg} \cdot \mathrm{L}^{-1}\right)$ & $280 \pm 240$ & $177 \pm 83$ & $142 \pm 111$ & $115 \pm 39$ & $99 \pm 45$ \\
\hline $\mathrm{SSV}\left(\mathrm{mg} \cdot \mathrm{L}^{-1}\right)$ & $241 \pm 195$ & $141 \pm 70$ & $123 \pm 92$ & $90 \pm 14$ & $79 \pm 34$ \\
\hline
\end{tabular}

( ) número de amostras e *DQO Filtrada

$\mathrm{CO}=$ carga orgânica aplicada/ciclo; $\mathrm{CS}=$ carga aplicada de sulfato/ciclo.

Adicionalmente realizaram-se exames microbiológicos por meio de Microscopia Ótica de Contraste de Fase e Fluorescência, nas amostras de biomassa aderidas no carvão mineral nos dois reatores ASBBR. Tais amostras foram visualizadas no microscópio OLYMPUS BX-60 com as imagens capturadas por câmera (OPTRONICS) acoplada ao software "Image Pro Plus" (versão 4.1). Para as amostras de agregados biológicos do ASBBR2, a fim de constatar a presença de enxofre (S) realizou-se no Instituto de Química (Universidade de São Paulo USP/São Carlos, SP), a análise por meio da técnica de Difração de Raios-X (EDX) com Microscópio Eletrônico de Varredura (Marca LEO) calibrado com cobalto.

Inoculo Anaeróbio (Inoculação)

Este procedimento tem a finalidade de permitir a aderência de biomassa ao meio suporte (carvão mineral). Nesta fase, os reatores ASBBR foram preenchidos com lodo anaeróbio (30\%) e para completar o volume líquido dos reatores $(70 \%)$ adicionou-se esgoto sanitário. A cada batelada ou ciclo, os reatores foram realimentados totalmente com esgoto sanitário e as bombas de recirculação foram mantidas acionadas. Esta operação durou 24 dias, ou seja, 12 ciclos de 48 h. Ressaltase que o lodo foi proveniente de reator UASB $\left(50 \mathrm{~m}^{3}\right)$ instalado na Estação de Tratamento de Esgotos Sanitários do Campus da Universidade de São Paulo (USP/São Carlos-SP). 


\section{RESULTADOS E DISCUSSÃO}

Os resultados médios das variáveis obtidas pelo reator ASBBR1 nos 92 ciclos operacionais para as várias Etapas de operação estão apresentados na Tabela 4.

Os dados obtidos em termos de redução de sulfato foram realmente expressivos ao longo da operação do reator ASBBR1. A eficiência média de redução de sulfato na Etapa I foi de 92\% (máx.: 99,8 \%) e na Etapa II de 88 \% (máx: 99,2\%), sendo que, no efluente do reator, nas respectivas Etapas, foram obtidos valores médios de sulfato de 0,021 $\pm 0,01$ g. $\mathrm{L}^{-1}$ (min: $1 \mathrm{mg} . \mathrm{L}^{-1}$ ) e de 0,064 $\pm 0,02 \mathrm{~g} / \mathrm{L}$ (min: $9 \mathrm{mg} . \mathrm{L}^{-1}$ ). Nas Etapas III, IV e $\mathrm{V}$, atingiram-se, respectivamente, os valores de $89 \%$ (máx.: 99,5\%), $87 \%$ (máx: 99,3\%) e $85 \%$ (máx: 99,1\%) em termos de eficiência média de remoção de sulfato. Os valores médios de sulfato no efluente foram 0,12 $\pm 0,05 \mathrm{~g} / \mathrm{L}$ (min: $9 \mathrm{mg} . \mathrm{L}^{-}$ ${ }^{1}$ ), 0,26 $\pm 0,07 \mathrm{~g} / \mathrm{L}$ (min: $9 \mathrm{mg} . \mathrm{L}^{-1}$ ) e de $0,48 \pm 0,18 \mathrm{~g} / \mathrm{L}$ (min: $14 \mathrm{mg} \cdot \mathrm{L}^{-1}$ ), respectivamente. A carga inicial aplicada de sulfato foi de $0,15 \mathrm{~kg} /$ ciclo e chegando a $1,9 \mathrm{~kg} / \mathrm{ciclo}$, sendo que a carga removida máxima foi de 1,6 kg/ciclo (Etapa V).

Tabela 4 - Resultados médios das variáveis obtidas pelo reator ASBBR1 nas várias Etapas de operação. $\mathrm{CO}$ = carga orgânica aplicada/ciclo; $\mathrm{CR}$ = carga removida de sulfato/ciclo.

\begin{tabular}{|c|c|c|c|c|c|}
\hline Parâmetros & Etapa I & Etapa II & Etapa III & Etapa IV & Etapa V \\
\hline Número de ciclos & $7(5)$ & $24(16)$ & $21(14)$ & $9(6)$ & $31(20)$ \\
\hline *Temperatura $\left({ }^{\circ} \mathrm{C}\right)$ & $24 \pm 3$ & $31 \pm 3$ & $30 \pm 3$ & $35 \pm 1$ & $34 \pm 1$ \\
\hline CR (kg/ciclo) & $0,15 \pm 0,01$ & $0,25 \pm 0,04$ & $0,60 \pm 0,07$ & $1,15 \pm 0,12$ & $1,60 \pm 0,21$ \\
\hline $\mathrm{SO}_{4}^{-2}\left(\mathrm{~g} . \mathrm{L}^{-1}\right)$ & $0,021 \pm 0,01$ & $0,064 \pm 0,02$ & $0,12 \pm 0,05$ & $0,26 \pm 0,07$ & $0,48 \pm 0,18$ \\
\hline **STD $\left(\mathrm{mg} . \mathrm{L}^{-1}\right)$ & $4,0 \pm 0,9$ & $25 \pm 8$ & $132 \pm 32$ & $221 \pm 71$ & $287 \pm 132$ \\
\hline $\mathrm{CO}(\mathrm{kg} / \mathrm{ciclo})$ & $0,09 \pm 0,03$ & $0,21 \pm 0,09$ & $0,45 \pm 0,12$ & $0,87 \pm 0,27$ & $1,80 \pm 0,56$ \\
\hline $\mathrm{DQO}_{\text {Total }}\left(\mathrm{g} \cdot \mathrm{L}^{-1}\right)$ & $0,14 \pm 0,03$ & $0,33 \pm 0,08$ & $0,72 \pm 0,16$ & $1,45 \pm 0,35$ & $3,01 \pm 0,57$ \\
\hline 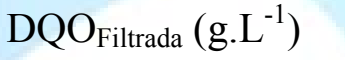 & $0,11 \pm 0,03$ & $0,24 \pm 0,07$ & $0,57 \pm 0,15$ & $1,07 \pm 0,33$ & $2,77 \pm 0,56$ \\
\hline $\mathrm{AB}\left(\mathrm{mgCaCO} 3 \cdot \mathrm{L}^{-1}\right)$ & $301 \pm 48$ & $471 \pm 50$ & $790 \pm 171$ & $1533 \pm 197$ & $765 \pm 772$ \\
\hline AVT (mgHac.L $\left.{ }^{-1}\right)$ & $24 \pm 20$ & $49 \pm 45$ & $172 \pm 63$ & $238 \pm 204$ & $1552 \pm 641$ \\
\hline $\operatorname{SST}\left(\mathrm{mg} \cdot \mathrm{L}^{-1}\right)$ & $35 \pm 24$ & $32 \pm 17$ & $46 \pm 32$ & $37 \pm 15$ & $40 \pm 12$ \\
\hline SSV (mg.L $\left.{ }^{-1}\right)$ & $25 \pm 20$ & $23 \pm 12$ & $38 \pm 33$ & $32 \pm 14$ & $27 \pm 12$ \\
\hline
\end{tabular}

( ) número de amostras, *líquido e $* *$ sulfetos totais dissolvidos

$\mathrm{CO}=$ carga orgânica aplicada/ciclo; $\mathrm{CR}=$ carga removida de sulfato/ciclo

As cargas orgânicas aplicadas variaram de 0,6 a 3,0 kg/ciclo nas várias Etapas. As eficiências médias de remoção de DQO foram superiores a 80\% nas Etapas I e II, decrescendo para valor médio de $41 \%$ na Etapa V. A baixa eficiência de remoção de matéria orgânica detectada nesse período específico deveu-se à presença de alta concentração de DQO residual, composta, principalmente, de ácidos voláteis totais 
(AVT) e de sulfetos totais dissolvidos (STD) gerados pela redução do sulfato (Tabela $4)$.

No que se refere à matéria orgânica solúvel, a alta concentração de ácidos orgânicos no efluente indicou que o processo metanogênico foi inibido, uma vez que este é o principal processo de consumo de ácidos orgânicos em reatores anaeróbios. Ressalta-se que a carga aplicada em termos de DQO foi gerada, principalmente, a partir da Etapa II, pela adição de etanol como doador de elétrons para o processo de redução do sulfato. $\mathrm{O}$ efluente do ASBBR1 atingiu médias para $\mathrm{DQO}_{\text {Bruta }}$ e $\mathrm{DQO}_{\text {Filtrada }}$ de $0,14 \pm 0,03$ g. $L^{-1}$ e de $0,11 \pm 0,03$ g. $L^{-1}$ na Etapa $I$ e de $0,33 \pm 0,08$ g. $L^{-1}$ e de $0,24 \pm 0,07$ g. $\mathrm{L}^{-1}$ na Etapa II, enquanto que nas Etapas III, IV e V estes valores foram de $0,72 \pm 0,16$ g. $L^{-1}$ e $0,57 \pm 0,15$ g. $L^{-1}, 1,45 \pm 0,35$ g. $L^{-1}$ e $1,07 \pm 0,33$ g. $L^{-1}$ e de $3,01 \pm 0,57$ g. $\mathrm{L}^{-1} \mathrm{e} 2,77 \pm 0,56 \mathrm{~g} . \mathrm{L}^{-1}$, respectivamente.

Nas Etapas I a IV, os valores de $\mathrm{pH}$ mantiveram-se entre 7,1 e 7,3. Especificamente na Etapa $\mathrm{V}$, as concentrações médias de sulfeto total dissolvido (STD) foram de $287 \pm 132 \mathrm{mg} . \mathrm{L}^{-1}$ (máx: $496 \mathrm{mg} . \mathrm{L}^{-1}$ ), sendo que o valor do $\mathrm{pH}$ no efluente decresceu de 7,3 (Etapa IV) para 6,7 (Etapa V), o que indicou maior concentração da forma de $\mathrm{H}_{2} \mathrm{~S}$ no meio líquido. $\mathrm{O}$ valor de $\mathrm{pH}$ foi influenciado pelo aumento da concentração de ácidos voláteis totais (AVT) produzidos pela oxidação incompleta do etanol para ácidos voláteis, principalmente, ácido acético. A presença de $\mathrm{H}_{2} \mathrm{~S}$, em alta concentração, pode ser considerada o fator que levou à inibição da atividade metanogênica no reator (LENS et al., 1998).

Somente na Etapa V foi observada a diminuição de $\mathrm{pH}$ para o valor de 6,7 (Tabela 4). Nessa Etapa, houve aumento exagerado na concentração de AVT presente no efluente (Figura 4) e, conseqüentemente, consumo proporcional de AB $(765 \pm 772$ $\left.\mathrm{mgCaCO}_{3} \cdot \mathrm{L}^{-1}\right)$. A presença de DQO residual no efluente, portanto, é explicada pela alta concentração de AVT presente $\left(1552 \pm 641 \mathrm{mgHac} . \mathrm{L}^{-1}\right)$ e fortalece a hipótese de supressão da metanogênese nesse período na presença de altas concentrações de sulfetos. O etanol oxidado parcialmente, porém, foi convertido a acetato, sendo pouco consumido pelos organismos metanogênicos e, portanto, descartado no efluente (DQO residual).

A geração de alcalinidade nos reatores foi crescente para as quatro primeiras Etapas, com valores médios de $\mathrm{AB}$ no efluente de $301 \pm 48 \mathrm{mgCaCO}_{3} \cdot \mathrm{L}^{-1}$ (Etapa I), $471 \pm 50 \mathrm{mgCaCO}{ }_{3} \mathrm{~L}^{-1}$ (Etapa II), $790 \pm 171 \mathrm{mgCaCO}_{3} \cdot \mathrm{L}^{-1}$ (Etapa III) e $1533 \pm 197$ $\mathrm{mgCaCO}_{3} \cdot \mathrm{L}^{-1}$ (Etapa IV). A produção de alcalinidade observada pode ser explicada pelo consumo de AVT até na Etapa IV. Os valores médios do efluente em termos de AVT nas Etapas I, II, III e IV foram de $24 \pm 20$ mgHac. $\mathrm{L}^{-1}, 49 \pm 45$ mgHac. $\mathrm{L}^{-1}, 172 \pm 63$ mgHac. $\mathrm{L}^{-1}$ e $238 \pm 204 \mathrm{mgHac} . \mathrm{L}^{-1}$, respectivamente.

$\mathrm{Na}$ Etapa I, o valor médio da relação $\mathrm{DQO} /\left[\mathrm{SO}_{4}{ }^{2-}\right]$ foi de 2,1 pela presença apenas de esgoto sanitário (Tabela 3). Nas Etapas II, III, IV e V, com a introdução de etanol, adequou-se a relação $\mathrm{DQO} /\left[\mathrm{SO}_{4}{ }^{2-}\right]$, a fim de alcançar as maiores eficiências de redução de sulfato. Os valores médios da relação $\mathrm{DQO} /\left[\mathrm{SO}_{4}{ }^{2-}\right]$ reduziram-se gradualmente com o aumento da concentração de sulfato para 1,9 (Etapa II), 1,8 
(Etapa III), 1,6 (Etapa IV) e 1,5 (Etapa V). Tais resultados indicaram que houve diminuição no consumo de etanol em função da carga removida $(\mathrm{CR})$ nas várias etapas de operação do ASBBR. Os valores obtidos de consumo de etanol (médio) foram de $1,60,1,35 ; 1,25$ e $1,1 \mathrm{~kg}$ etanol $/ \mathrm{kg}$ sulfato removido/ciclo. Considerou-se densidade do etanol combustível de $810 \mathrm{~kg} \cdot \mathrm{m}^{-3}$.

O consumo de etanol é importante no processo de redução de sulfato, já que a sua aplicação envolve o custo do produto. Como o etanol utilizado é o mesmo vendido em postos, o preço por litro está em torno de R \$1,30. Na Etapa I, o esgoto sanitário foi adicionado como matéria orgânica, portanto, a custo zero. Para a Etapa $\mathrm{V}$, ao remover-se a carga de sulfato de $1,6 \mathrm{~kg} /$ ciclo, o custo calculado foi de $\mathrm{R} \$$ 2,52/ciclo, ou seja, $\mathrm{R} \$ 1,57 / \mathrm{kg}$ sulfato removido.

O ASBBR2 foi monitorado durante as Etapas IV e $\mathrm{V}$ de operação do reator ASBBR1. Na Tabela 4 apresenta-se as características do afluente aplicadas ao reator utilizado como unidade de pós-tratamento. Na Tabela 5 estão apresentados os resultados experimentais obtidos pelo monitoramento da unidade. Ressalta-se que a variação das concentrações de ácidos voláteis totais e de sulfeto dissolvido foi pequena, mesmo com o incremento da concentração de sulfato e, conseqüentemente, de etanol no reator ASBBR1. Na verdade ocorreu menor consumo, principalmente, de etanol quando a concentração de sulfato foi aumentada nesse sistema (2,0 para 3,0 g. $\mathrm{L}^{-1}$ ) e assim gerando valores de ácidos voláteis totais bem próximos.

Ao longo da operação do reator ASBBR2, foram obtidos resultados expressivos em termos de redução de DQO, sendo que as cargas orgânicas aplicadas variaram de 0,66 a $0,91 \mathrm{~kg} /$ ciclo. As eficiências de remoção chegaram a valores superiores a $85 \%$, tanto nas amostras brutas como nas filtradas, e concentrações no efluente em torno de $300 \mathrm{mg} . \mathrm{L}^{-1}$ (amostras brutas e filtradas).

No ASBBR2 para pós-tratamento, a eficiência de remoção de matéria orgânica via processo anaeróbio esteve relacionada diretamente na conversão dos ácidos voláteis gerados no processo de redução de sulfato para metano, já que na sua totalidade está na forma de ácido acético. Pode-se verificar consumo efetivo de AVT, e consequentemente a redução da DQO residual (matéria orgânica) efluente (Tabelas 4 e 5). O consumo de AVT refletiu na geração de alcalinidade a bicarbonato. Portanto, etanol oxidado parcialmente e convertido preferencialmente em ácido acético foi quase totalmente consumido pelos organismos metanogênicos nessa unidade. As observações microbiológicas dos microrganismos aderidos demonstraram a clara presença de arqueas metanogênicas, principalmente, semelhantes à Methanosaeta sp. (Figura 2). 
Tabela 5 - Resultados médios das variáveis obtidas pelo reator ASBBR2 nos 35 ciclos de operação. $\mathrm{CO}=$ carga orgânica aplicada/ciclo.

\begin{tabular}{|c|c|c|c|}
\hline Parâmetros & Mínimo & Máximo & Média \\
\hline Temperatura líquido $\left({ }^{\circ} \mathrm{C}\right)$ & 29 & 35 & $32 \pm 3$ \\
\hline $\mathrm{pH}$ & 7,2 & 7,9 & - \\
\hline $\mathrm{AB}\left(\mathrm{mgCaCO}_{3}{ }^{-2} \cdot \mathrm{L}^{-1}\right)$ & 1074 & 1550 & $1350 \pm 118$ \\
\hline $\operatorname{AVT}\left(\mathrm{mgHac} . \mathrm{L}^{-1}\right)$ & 22 & 232 & $67 \pm 23$ \\
\hline $\mathrm{DQO}_{\text {Total }}\left(\mathrm{mg} \cdot \mathrm{L}^{-1}\right)$ & 239 & 496 & $386 \pm 66$ \\
\hline CO (kgDQO/ciclo) & 0,66 & 0,91 & - \\
\hline $\mathrm{COD}_{\text {Filtered }}\left(\mathrm{mg} \cdot \mathrm{L}^{-1}\right)$ & 111 & 375 & $276 \pm 58$ \\
\hline $\mathrm{SO}_{4}^{-2}\left(\mathrm{mg} \cdot \mathrm{L}^{-1}\right)$ & 2 & 8 & $5 \pm 2$ \\
\hline STD (mg.L $\left.L^{-1}\right)$ & 5 & 130 & $66 \pm 19$ \\
\hline $\mathrm{SST}\left(\mathrm{mg} \cdot \mathrm{L}^{-1}\right)$ & 24 & 66 & $34 \pm 11$ \\
\hline $\mathrm{SSV}\left(\mathrm{mg} \cdot \mathrm{L}^{-1}\right)$ & 21 & 40 & $28 \pm 6$ \\
\hline
\end{tabular}

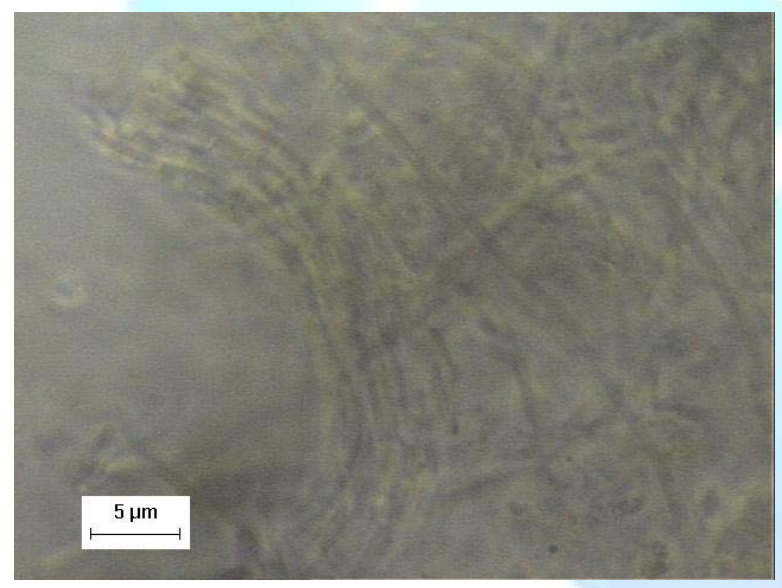

(a)

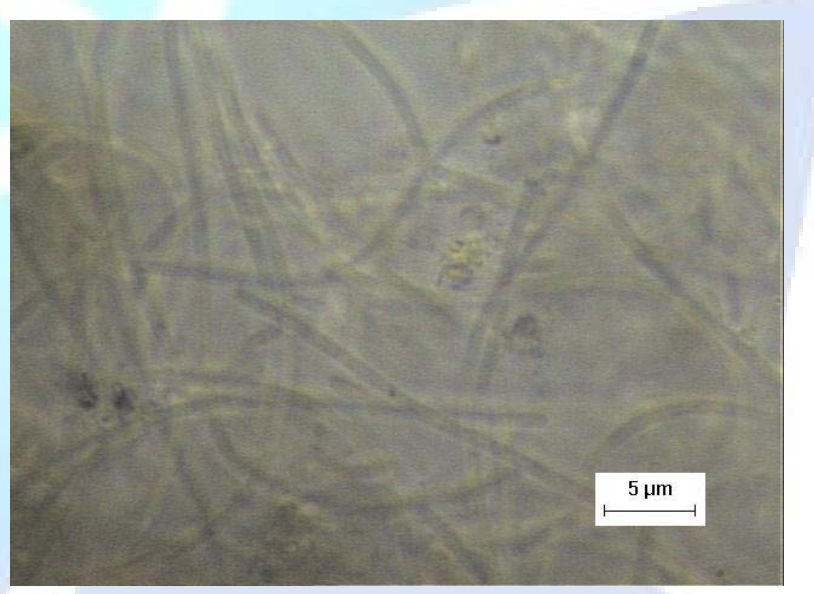

(b)

Figura 2. Morfologias observadas por exame microscópico em contraste de fase das células aderidas no carvão mineral provenientes do reator ASBBR2. Estruturas semelhantes $a:(a, b)$ arquea filamentosa como Methanosaeta sp (aumento 1500X).

Pelo monitoramento do ASBBR2 a efetiva geração de alcalinidade a bicarbonato via processo anaeróbio obteve-se o valor médio de $1352 \pm 118$ $\mathrm{mgCaCO}_{3} \cdot \mathrm{L}^{-1}$ (máx.: $1550 \mathrm{mgCaCO}_{3} \cdot \mathrm{L}^{-1}$ ). Logicamente, a produção de alcalinidade pode explicar as baixas concentrações de AVT. No afluente foi obtido valor médio em termos de AVT de 1552 \pm 641 mgHac.L ${ }^{-1}$ (máx.: 1645 mgHac. $\mathrm{L}^{-1}$ ) e no efluente de 
$67 \pm 23$ mgHac. $\mathrm{L}^{-1}$ (min.: $22 \mathrm{mgHac} . \mathrm{L}^{-1}$ ). $\mathrm{O}$ pH no efluente ficou na faixa de 7,2 e 7,9, enquanto no afluente com valores de 5,4 a 6,2.

Nesta pesquisa, a intenção maior foi avaliar a conversão da matéria orgânica conjuntamente com a redução da concentração de sulfetos totais (STD). Os dados de remoção de STD foram reduzidos e chegou-se ao final da fase experimental com valor médio de 57\%. As concentrações STD no efluente tiveram valor médio de $66 \pm 19 \mathrm{mg} . \mathrm{L}^{-1}$. Salienta-se que as concentrações de sulfato no afluente e efluente mantiveram-se abaixo de $8 \mathrm{mg} . \mathrm{L}^{-1}$.

Acredita-se que este sulfeto foi removido na sua maior parte na forma de enxofre elementar. Para isso, conforme o trabalho de Janssen et al., 1998, as condições são especificas para a sua obtenção, tais como baixa concentração de OD $\left(<0,1 \mathrm{mgO}_{2} \cdot \mathrm{L}^{-1}\right)$ e potencial redox (POR) em torno de $-150 \mathrm{mV}$. Evidenciou na operação da unidade a formação na superfície líquida de quantidades apreciáveis de agregados biológicos de cor amarelo palha ao final dos ciclos, o que indicaria a presença de enxofre elementar, mesmo com resultados de POR de $-350 \mathrm{mV}$ (anaerobiose estrita). O resultado da técnica de difração de Raio X de amostra seca dos agregados biológicos, principalmente, para o elemento enxofre (s) está apresentado na Figura 3.

O processo de conversão de sulfetos para enxofre elementar ocorreu provavelmente pela oxidação biológica ocorrida pela presença de oxigênio atmosférico na superfície líquida. A formação de agregados biológicos permite que seja possível a separação do meio líquido. Para tanto, instalou-se sedimentador (Figura 1a) para a separação do meio líquido. A final do ciclo, cerca de $50 \mathrm{~L}$, eram removidos antes do descarte final a fim de recolher os agregados e propiciar sua sedimentação. Os resultados foram poucos satisfatórios em virtude da não ocorrência da esperada sedimentação total. Parcela considerável dos agregados biológicos manteve-se na superfície líquida do sedimentador. Observações microbiológicas realizadas da amostra do sólido removido da superfície líquida revelaram a presença do enxofre elementar com aspecto amarelo brilhante e presença de grande quantidade de bacilos (Figura 3a e b) formando agregados biológicos. 


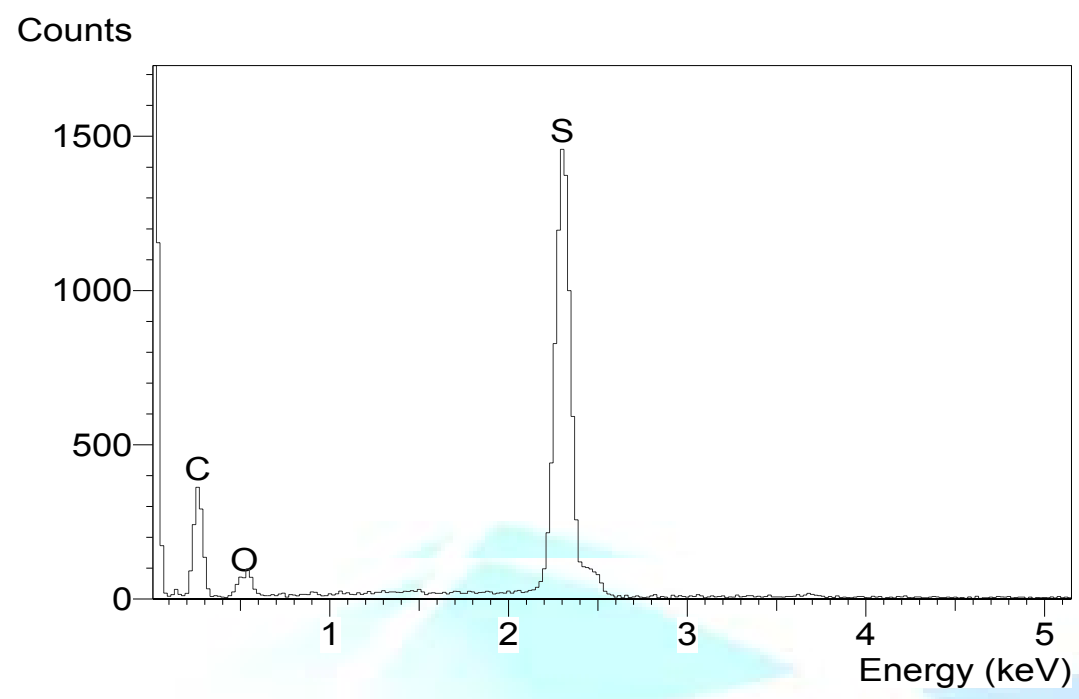

Figura 3. Resultado da detecção de enxofre (S) por meio de EDX.

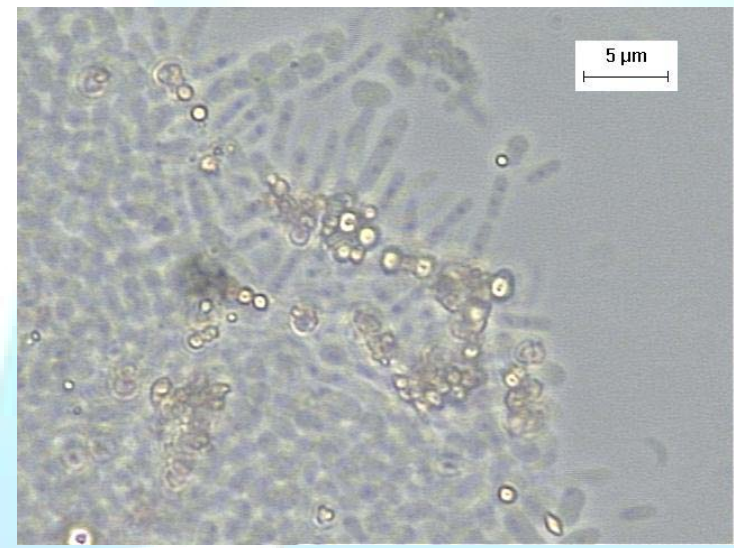

(a)

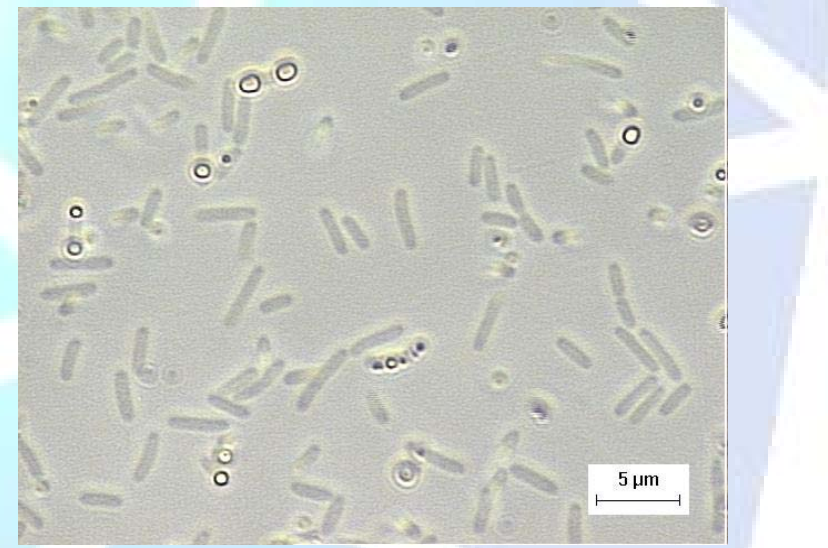

(b)

Figura 4. Morfologias observadas por exame microscópico em contraste de fase de amostras provenientes da superfície líquida do ASBBR2 com aumento de 1500X. Estruturas semelhantes a: (a) bacilos coloniais e enxofre elementar; (b) bacilos de bordas arredondadas com inclusões de enxofre.

\section{CONCLUSÕES}

A aplicação de tratamento biológico em efluente industrial contendo elevadas concentrações de sulfato $\left(0,25 ; 0,50 ; 1,0 ; 2,0\right.$ e 3,0 g. $\left.\mathrm{L}^{-1}\right)$ forneceu resultados expressivos em termos de redução de sulfato (88 a 92\%), o que permitiu vislumbrar a possibilidade de uso de reator anaeróbio ASBBR1 para tal finalidade e, no futuro, a aplicação em larga escala no tratamento de outras águas residuárias ricas em sulfato. 
Os resultados de desempenho do ASBBR1 demonstraram o potencial desta configuração de reator, não somente para a remoção de sulfato, mas para a remoção conjunta de matéria orgânica e sulfato em faixas apreciáveis para valores de até 2,0 g.L $L^{-1}$ (Etapa IV). Tais valores permitiram a adequação do efluente do ASBBR ao padrão de emissão do íon sulfato no Estado de São Paulo (<.1,0 g.. $\left.\mathrm{L}^{-1}\right)$.

Constatou-se, no entanto, a formação de altas concentrações de compostos reduzidos de enxofre (STD) e de DQO residual para concentrações de sulfato superiores a 2,0 g.L $\mathrm{L}^{-1}$. Por essa razão, a aplicação desse processo em escala industrial torna necessária a implantação de sistema de pós-tratamento para adequar os efluentes aos padrões de emissão. A remoção da DQO residual composta essencialmente por ácidos orgânicos, tais como, ácido acético é relativamente simples podendo ser utilizado reatores biológicos anaeróbios.

A remoção de compostos reduzidos de enxofre, principalmente dos efluentes líquidos, deve ser concebida de maneira a tornar possível a produção de enxofre elementar, de maneira a direcionar os sistemas de tratamento para recuperação de subprodutos e assim torná-los mais sustentáveis do ponto de vista ambiental e econômico. Na forma de enxofre elementar existe a viabilidade de retorno a cadeia produtiva (ácido sulfúrico), bem como condicionante de solo.Neste sentido foi obtida remoção média de DQO residual superior $85 \%$ no ASBBR2 concebido para tal finalidade. Em conjunto foi possível reduzir em média $57 \%$ dos sulfetos totais presentes no meio líquido. Este dado de eficiência não nos permitiu adequar este efluente para descarte, já que as concentrações devem ficar abaixo de $1,0 \mathrm{mg} . \mathrm{L}^{-1}$.

Assim sendo, o foco principal foi apontar uma solução possível para a remoção biológica do sulfato e seus subprodutos utilizando tecnologia e materiais disponíveis no país. Novos estudos necessitam ser desenvolvido enfocando diretamente o póstratamento, bem como a recuperação do enxofre elementar.

\section{AGRADECIMENTOS}

À Fundação de Amparo à Pesquisa do Estado de São Paulo (FAPESP - Processo no 03/07799-2) e ao Conselho Nacional de Desenvolvimento Científico e Tecnológico (Edital Universal $n^{0}$ 019/2004 - Processo $n^{0}$ 478355/2004-1) pelo apoio financeiro na execução da pesquisa.

\section{REFÊRENCIAS}

APHA, AWWA, WPCF. Standard Methods for the examination of water and wastewater, $19^{\text {th }}$ edition, American Public Health Association, Washington, 1998. 
COLLERAN, E.; FINNEGAN, S.; LENS, P. Anaerobic treatment of sulphatecontaining waste streams. Departament of Microbiology, University College, Galway, Republic of Ireland, 1994.

DILLALO, R; ALBERTSON, O.E. Volatile acids by direct tritation. Journal WPCF. v.33, p.356-365, 1961.

HULSHOFF-POL, L.W.; LENS, P.N.L.; WEIJMA, J.; STAMS A.J.M. New developments in reactor and process technology for sulfate reduction. Water Science and Technology, v. 44 (8), p.67-76, 2001.

JANSSEN, A. J. H.; MEIJER, S.; BONTSEMA, J.; LETTINGA,G. Application of the redox potential for controling a sulfide oxidizing bioreactor. Biotechnology and Bioengineering. v.60, p.147-155, 1998.

LENS, P.N.L.; VISSER A.; JANSSEN, A.J.H.; HULSHOFF POL, L.W.; Lettinga G. Biotechnological treatment of sulfate-rich wastewaters. Critical Reviews in Environmental Science and Technology, v.28 (1), p.41-88, 1998.

NAGPAL, S.; CHUICHULCHERM, S.; PEEVA L.; LIVINGSTON, A. Microbial sulfate reduction in a liquid-solid fluidized bed reactor. Biotechnology Bioengineering, v.70 (4), p.370-379, 2000.

RIPLEY, L.E.; BOYLE, W.C.; Converse, J.C. Improved alkalimetric monitoring for anaerobic digestion of high-strength wastes. Journal WPCF. v.58, p.406-411, 1986.

SARTI, A.; SILVA, A.J.; CÔRTES, R.S.; FORESTI, E. Remoção de sulfato de águas residuárias industriais em reator anaeróbio de leito fixo operado em bateladas seqüenciais. Engenharia Sanitária e Ambiental (ABES), v.13, p.15-22, 2008.

SILVA, A.J.; VARESCHE, M.B.; FORESTI E.; ZAIAT, M. Sulphate removal from industrial wastewater using a packed-bed anaerobic reactor. Process Biochemistry, v.37, p.927-935, 2002.

VAN HOUTEN, R.T.; VAN DER SPOEL, H.; VAN AELST, A.; HULSHOFF POL, L.W.; LETTINGA, G. Biological sulphate reduction using synthesis gas as energy and carbon source. Biotechnology and Bioengineering, v.50, p.136-144, 1996. 